Pacific Journal of Mathematic 


\title{
ON THE NIELSEN NUMBER OF A FIBER MAP
}

\author{
Dennis McGavran and Jingyal PaK
}

Suppose $\mathscr{T}=\{E, \pi, B, F\}$ is a fiber space such that $0 \rightarrow$ $\pi_{1}(F) \stackrel{i_{\sharp}}{\rightarrow} \pi_{1}(E) \stackrel{\pi_{\#}}{\rightarrow} \pi_{1}(B) \rightarrow 0$ is exact. Suppose also that the above fundamental groups are abelian. If $f: E \rightarrow E$ is a fiber preserving map such that $f_{\sharp}(\alpha)=\alpha$ if and only if $\alpha=0$, then it is shown that $R(f)=R\left(f^{\prime}\right) \cdot R\left(f_{b}\right)$ where $R(h)$ is the Reidemeister number of the map $h$.

A product formula for the Nielsen number of a fiber map which holds under certain conditions was introduced by $R$. Brown. Let $\mathscr{T}=\left\{E, \pi, L,(p, q), s^{1}\right\}$ be a principal $s^{1}$-bundle over the lens space $L(p, q)$, where $\mathscr{T}$ is determined by $\left[f_{j}\right] \epsilon$ $\left[L(p, q), c p^{\infty}\right] \simeq H^{2}(L(p, q), z) \simeq z_{p}$. Let $f: E \rightarrow E$ be a fiber preserving map such that $f_{b_{\sharp}}(1)=c_{2}, f_{\sharp}^{\prime}\left(\bar{l}_{p}\right)=\bar{c}_{1}$, where 1 generates $\pi_{1}\left(s^{1}\right) \simeq z$ and $\bar{l}_{p}$ generates $\pi_{1}(L(p, q)) \simeq z_{p}$. Then the Nielsen numbers of the maps involved satisfy

$$
N(f)=N\left(f_{b}\right) \cdot\left(d, 1-c_{1}, s\right),
$$

where $d=(j, p)$ and $s=j / p\left(c_{1}-c_{2}\right)$.

I. Introduction. Let $\mathscr{T}=\{E, \pi, B, F\}$ be a fiber space. Any fiber preserving map $f: E \rightarrow E$ induces maps $f^{\prime}: B \rightarrow B$, and, for each $b \in B, f_{b}: \pi^{-1}(b) \rightarrow \pi^{-1}(b)$, where $\pi^{-1}(b) \simeq F$. The map $f$ will be called a fiber map (or bundle map if $\mathscr{T}$ is a bundle).

Let $N(g)$ denote the Nielsen number of a map $g$. The Nielsen number, $N(g)$, serves as a lower bound on the number of fixed points of a map homotopic to $g$, and under certain hypotheses, there exists a map homotopic to $g$ with exactly $N(g)$ fixed points. R. Brown and E. Fadell ([2] and [3]) proved the following:

THEOREM. Let $\mathscr{T}=\{E, \pi, B, F\}$ be a locally trivial fiber space, where $E, B$, and $F$ are connected finite polyhedra. Let $f: E \rightarrow E$ be a fiber map. If one of the following conditions holds:

(i) $\pi_{1}(B)=\pi_{2}(B)=0$.

(ii) $\pi_{1}(F)=0$.

(iii) $\mathscr{T}$ is trivial and either $\pi_{1}(B)=0$ or $f=f^{\prime} \times f_{b}$ for all $b \in B$ then $N(f)=N\left(f^{\prime}\right) \cdot N\left(f_{b}\right)$ for all $b \in B$.

These strong restrictions on the spaces involved eliminate some interesting fiber spaces. For example, any circle bundle over $B$ with $\pi_{1}(B) \neq 0$ is excluded. Furthermore, if $\pi_{1}(B)=\pi_{2}(B)=0$, then the total space $E$ is $B \times S^{1}$.

This paper has two objectives. The first is to try to generalize 
the above result to the case of a bundle $\mathscr{T}=\{E, \pi, B, F\}$ where $\pi_{1}(B)$ is a nontrivial abelian group, and $\pi_{2}(B)=0$. The second is to investigate the relationships between the Nielsen numbers of the maps $f, f^{\prime}$, and $f_{b}$ for particular circle bundles.

In this paper all spaces are path-connected.

II. Some general results. The reader may refer to [1] and [2] for definitions and details concerning the Nielsen number $N(f)$, Reidemeister number $R(f)$, and Jiang subgroup $T(f)$ of a map $f: X \rightarrow X$.

We will be particularly interested in the Reidemeister number. It serves as an upper bound on $N(f)$ and in many cases $R(f)=N(f)$. Let $h: G \rightarrow G$ be a homomorphism where $G$ is an abelian group. It is shown in [1] that $R(h)=|\operatorname{coker}(1-h)|(||$ means the order of a group). The Reidemeister number of a map $f: X \rightarrow X$ is defined to be the Reidemeister number of the induced homomorphism $f_{\sharp}: \pi_{1}(X) \rightarrow$ $\pi_{1}(X)$. Now let $\mathscr{T}$ be a fiber space. Let $F_{b}=\pi^{-1}(b)$. If $w: I \rightarrow B$ is such that $w(0)=b$ and $w(1)=b^{\prime}$, we may translate $F_{b^{\prime}}$ along the path $w$ to $F_{b}$ (see [6]). This gives a homeomorphism $\bar{w}: F_{b^{\prime}} \rightarrow F_{b}$. Given a fiber map $f: E \rightarrow E$, we have the natural map $f_{b}^{\prime \prime}: F_{b} \rightarrow F_{f^{\prime}(b)}$, the restriction of $f$ to $F_{b}$. Then by definition $f_{b}=\bar{w} \circ f_{b}^{\prime \prime}$. For more details on $f_{b}: F_{b} \rightarrow F_{b}$ readers are referred to [2].

Suppose $\mathscr{T}$ is a fiber space and $w$ is a loop based at $b$. Then we have $\bar{w}: \pi^{-1}(b) \rightarrow \pi^{-1}(b)$. The fiber space $\mathscr{T}$ is said to be orientable if the induced homomorphism $\bar{w}_{*}: H_{*}\left(\pi^{-1}(b), z\right) \rightarrow H_{*}\left(\pi^{-1}(b), z\right)$ is the identity homomorphism for every loop $w$ based at $b$. It is shown in [2] that if $\mathscr{T}$ is orientable and if the Jiang subgroup $T\left(p^{-1}(b), e_{0}\right)=$ $\pi_{1}\left(p^{-1}(b), e_{0}\right)$ for a fixed $b \in B$ then the Nielsen number of $f_{b}$ is independent of the choice of path from $f^{\prime}(b)$ to $b$. Furthermore, the Nielsen number $N\left(f_{b}\right)$ is independent of the choice of $b \in B$.

LemMa 1. Let $\mathscr{T}$ be a fiber space with $\pi_{1}(F), \pi_{1}(E)$, and $\pi_{1}(B)$ abelian. Suppose $f: E \rightarrow E$ is a fiber map. Then the following diagram commutes:

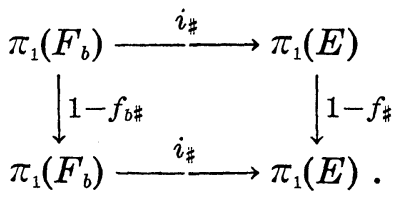

Proof. First, by [6], the map $\bar{w}$ is homotopic in $E$ to the identity map on $F_{f^{\prime}(b)}$. Hence we have

$$
\begin{aligned}
i_{\sharp} \circ\left(1-f_{b \sharp}\right)(\alpha) & =i_{\sharp}\left[\alpha-\left(\bar{w} \circ f_{b}^{\prime \prime}\right)_{\sharp}(\alpha)\right] \\
& =i_{\sharp}(\alpha)-i_{\sharp}\left(\bar{w} \circ f_{b}^{\prime \prime}\right)_{\sharp}(\alpha)=i_{\sharp}(\alpha)-\left(i_{\sharp} \circ f_{\sharp \sharp}^{\prime \prime}\right)(\alpha) \\
& =i_{\sharp}(\alpha)-\left(f_{\sharp} \circ i_{\sharp}\right)(\alpha)=\left(1-f_{\sharp}\right) \circ i_{\sharp}(\alpha) .
\end{aligned}
$$


Lemma 2 [4]. Suppose we have the following commutative diagram of modules, where the rows are exact:

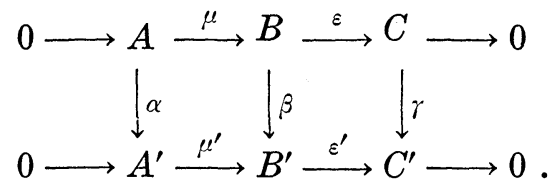

Then there is an exact sequence

$$
\begin{aligned}
& 0 \longrightarrow \operatorname{ker} \alpha \stackrel{\mu_{*}}{\longrightarrow} \operatorname{ker} \beta \stackrel{\varepsilon_{*}}{\longrightarrow} \operatorname{ker} \gamma \\
& \stackrel{\omega}{\longrightarrow} \operatorname{coker} \alpha \stackrel{\mu_{*}^{\prime}}{\longrightarrow} \operatorname{coker} \beta \stackrel{\varepsilon_{*}^{\prime}}{\longrightarrow} \operatorname{coker} \gamma \longrightarrow 0 .
\end{aligned}
$$

The homomorphisms $\mu_{*}$ and $\varepsilon_{*}$ are restrictions of $\mu$ and $\varepsilon$, and $\mu_{*}^{\prime}$ and $\varepsilon_{*}^{\prime}$ are induced by $\mu^{\prime}$ and $\varepsilon^{\prime}$ on quotients. The connecting homomorphism $\omega: \operatorname{ker} \gamma \rightarrow \operatorname{coker} \alpha$ is defined as follows. Let $c \in \operatorname{ker} \gamma$, choose $b \in B$ with $\varepsilon b=c$. Since $\varepsilon^{\prime} \beta b=\gamma \varepsilon b=\gamma c=0$ there exists $a^{\prime} \in A^{\prime}$ with $\beta b=\mu^{\prime} a^{\prime}$. Define $\omega(c)=\left[\alpha^{\prime}\right]$, the coset of $a^{\prime}$ in coker $\alpha$. Then $\omega$ is a well-defined homomorphism. See [4, p. 99] for the proof of the lemma.

Theorem 3. Suppose $\mathscr{T}=\{E, \pi, B, F\}$ is a fiber space such that

$$
0 \longrightarrow \pi_{1}(F) \stackrel{i_{\sharp}}{\longrightarrow} \pi_{1}(E) \stackrel{\pi_{\sharp}}{\longrightarrow} \pi_{1}(B) \longrightarrow 0
$$

is an exact sequence of abelian groups. Suppose $f: E \rightarrow E$ is a fiber map and $w: I \rightarrow B$ is a path from $b$ to $f^{\prime}(b)$. Then we have the following exact sequence:

$$
\begin{aligned}
0 \longrightarrow \operatorname{ker}\left(1-f_{b \sharp}\right) \longrightarrow \operatorname{ker}\left(1-f_{\sharp}\right) \longrightarrow \operatorname{ker}\left(1-f_{\sharp}^{\prime}\right) \\
\longrightarrow \operatorname{coker}\left(1-f_{b \sharp}\right) \longrightarrow \operatorname{coker}\left(1-f_{\sharp}\right) \longrightarrow \operatorname{coker}\left(1-f_{\sharp}^{\prime}\right) \longrightarrow 0 .
\end{aligned}
$$

Proof. The fiber map induces the following commutative diagram:

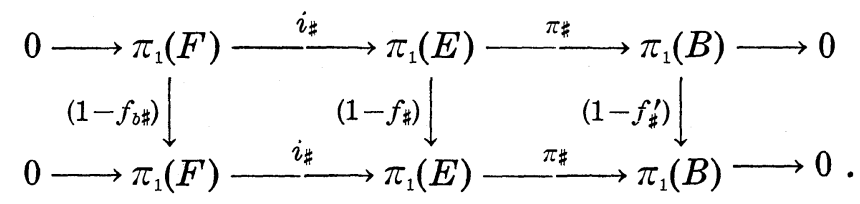

Now the result becomes a simple application of Lemmas 1 and 2 .

COROLlary 4. $\operatorname{ker}\left(1-f_{b \ddagger}\right)$ is independent of $w$ and $b$.

Proof. $\operatorname{ker}\left(1-f_{b \sharp}\right)$ is isomorphic to the kernel of the map $\operatorname{ker}\left(1-f_{\sharp}\right) \stackrel{\pi_{\sharp *}}{\longrightarrow} \operatorname{ker}\left(1-f_{\sharp}^{\prime}\right)$. But this map is the restriction of $\pi_{\ddagger}: \pi_{1}(E) \rightarrow \pi_{1}(B)$, which is independent of $w$ and $b$. 
Suppose $h: G \rightarrow G$ is a homomorphism of abelian groups. We will say that $h$ satisfies Condition $\mathrm{A}$ if $h(\alpha)=\alpha$ if and only if $\alpha=0$.

THEOREM 5. Suppose $\mathscr{T}$ is a fiber space satisfying the hypotheses of Theorem 3. Suppose $f: E \rightarrow E$ is a fiber map such that $f_{\sharp}^{\prime}$ satisfies Condition A. Then $R(f)=R\left(f^{\prime}\right) \cdot R\left(f_{b}\right)$ for all $b \in B$.

Proof. We have $\left(1-f_{\sharp}^{\prime}\right)(\alpha)=0$ if and only if $f_{\sharp}^{\prime}(\alpha)=\alpha$ if and only if $\alpha=0$. Therefore, $1-f_{\sharp}^{\prime}$ is injective and we have the following exact sequence:

$$
0 \rightarrow \operatorname{coker}\left(1-f_{b \sharp}\right) \rightarrow \operatorname{coker}\left(1-f_{\sharp}\right) \rightarrow \operatorname{coker}\left(1-f_{\sharp}^{\prime}\right) \rightarrow 0 .
$$

The theorem follows from the properties of $R(f)$.

COROLLARY 6. Under the hypotheses of Theorem $5 R\left(f_{b}\right)$ is independent of $w$ and $b$.

Proof. This follows since both $R(f)$ and $R\left(f^{\prime}\right)$ are independent of $w$ and $b$.

ExAmple 1. Let $\mathscr{T}$ be a principal $T^{k}$-bundle over a $(2 n+1)$ dimensional lens space $L(p), p \geqq 1$. We know from [5] that $L=$ $L(d) \times T^{k}$ where $d$ divides $p$. Let $f: E \rightarrow E$ be a bundle map. It follows easily from results in [1] that $N\left(f_{b}\right)=R\left(f_{b}\right)$. It is also shown in [1] that $N\left(f^{\prime}\right)=R\left(f^{\prime}\right)$ for $n=1$, and the proof can be easily generalized to higher dimensions. Furthermore, by showing that $T(f)=\pi_{1}\left(L(d) \times T^{k}\right)$, where $T(f)$ is the Jiang subgroup of $f$, one can show that $N(f)=R(f)$. Now such a bundle satisfies the hypothesis of Theorem 3. Hence, if $f_{\sharp}^{\prime}: \pi_{1}(L(p)) \rightarrow \pi_{1}(L(p))$ satisfies the hypothesis of Theorem 5, we have $N(f)=N\left(f^{\prime}\right) \cdot N\left(f_{b}\right)$ for all $b \in B$.

EXAMPLE 2. If $G$ is a compact connected semi-simple Lie group, then $\mathscr{T}=\left\{E, \pi, G, S^{1}\right\}$ satisfies the hypothesis of Theorem 3 . If $f: E \rightarrow E$ is a fiber map then $N(f)=N\left(f^{\prime}\right) \cdot N\left(f_{b}\right)$ follows from [3] since the second integral cohomology group of $G$ vanishes. Assume $N\left(f^{\prime}\right) \neq 0 \neq N\left(f_{b}\right)$. Then since $G$ and $S^{1}$ are $H$-spaces $T\left(f^{\prime}\right)=\pi_{1}(G)$ and $T\left(f_{b}\right)=\pi_{1}\left(S^{1}\right)$; and we have $N\left(f^{\prime}\right)=R\left(f^{\prime}\right)$ and $N\left(f_{b}\right)=R\left(f_{b}\right)$. It follows that $R(f)=R\left(f^{\prime}\right) \cdot R\left(f_{b}\right)$ independent of Condition A.

Lemma 7. Suppose $h: Z_{p} \rightarrow Z_{p}$ is such that $h(\bar{l})=\bar{m}$. Then Condition A holds iff $(1-m, p)=1$.

Proof. Suppose $(1-m, p)=1$. If $h(\bar{n})=\bar{m} \bar{n}=\bar{n}, 1 \leqq n<p$, then $m n \equiv n(\bmod p)$. Hence $p$ divides $(1-m) n$, which is impossible if $(1-m, p)=1$. 
Now suppose $h(\alpha)=\alpha$ iff $\alpha=0$. Suppose $(1-m, p)=d$. Let $1-m=c_{1} d, p=c_{2} d$. Then $h\left(\bar{c}_{2}\right)=\bar{m} \bar{c}_{2}$. Now

$$
m c_{2}-c_{2}=c_{2}(m-1)=-c_{2} c_{1} d=-c_{1} p .
$$

Thus $h\left(\bar{c}_{2}\right)=\bar{c}_{2}$ and $d=1$.

EXAMPLE 1 (con't). We have $\pi_{1}(L(p)) \simeq Z_{p}$. Suppose $f_{\sharp}^{\prime}(\bar{l})=\bar{m}$. Then $N\left(f^{\prime}\right)=(1-m, p)$. Hence Theorem 5 is applicable if and only if $N\left(f^{\prime}\right)=1$.

III. A general solution to Example 1. Let $\mathscr{T}=\left\{E, \pi, L(p, q), s^{1}\right\}$ be a principal $s^{1}$-bundle over a 3 -dimensional lens space $L(p, q)$. If $\mathscr{T}$ is induced by $\left[f_{j}\right] \in\left[L(p, q), C P^{\infty}\right] \simeq H^{2}(L(p, q), Z) \simeq Z_{p}$, then $E \simeq$ $L(d, q) \times s^{1}$, where $d=(j, p)$ (see [7]). Let $j=j^{\prime} d, p=p^{\prime} d$.

THEOREM 8. Let $\mathscr{T}$ be as above and $f: E \rightarrow E$ a fiber map such that, for a particular choice of $b \in B$ and $w, f_{b \ddagger}(1)=c_{2}$ and $f_{\sharp}^{\prime}\left(\bar{l}_{p}\right)=\bar{c}_{1}$, where 1 generates $\pi_{1}\left(s^{1}\right) \simeq Z$ and $\bar{l}_{p}$ generates $\pi_{1}(L(p, q)) \simeq Z_{p}$. Let $s=j / p\left(c_{1}-c_{2}\right)$. Then

$$
N(f)=N\left(f_{b}\right) \cdot\left(d, 1-c_{1}, s\right) .
$$

Proof. We first examine the structure of $L(d, q) \times s^{1}$ as an $s^{1}$-bundle over $L(p, q)$ (see [7]). $L(p, q)$ and $L(d, q)$ are obtained from $s^{3}$ as the orbit space of a free $Z_{p}$-action and $Z_{d}$-action, respectively. Given $\left(\left(r_{1}, \theta_{1}\right),\left(r_{2}, \theta_{2}\right)\right) \in s^{3}$, let $\left\langle\left(r_{1}, \theta_{1}\right),\left(r_{2}, \theta_{2}\right)\right\rangle$ represent its equivalence class as an element in $L(p, q)$. In $L(d, q) \times I, I=[0,2 \pi]$, identify $\left\{\left\langle\left(r_{1}, \theta_{1}\right),\left(r_{2}, \theta_{2}\right)\right\rangle, 2 \pi\right\}$ with $\left\{\left\langle\left(r_{1}, \theta_{1}+j^{\prime} v\right),\left(r_{2}, \theta_{2}+j^{\prime} q v\right)\right\rangle, 0\right\}$ to obtain $E$, where $v=2 \pi / p$. Define $h: E \rightarrow L(d, q) \times S^{1}$ by

$$
h\left\{\left\langle\left(r_{1}, \theta_{1}\right),\left(r_{2}, \theta_{2}\right)\right\rangle, t\right\}=\left\{\left\langle\left(r_{1}, \theta_{1}+\frac{t}{2 \pi} j^{\prime} v\right),\left(r_{2}, \theta_{2}+\frac{t}{2 \pi} j^{\prime} q v\right)\right\rangle, t\right\} .
$$

Then $h$ is a homeomorphism. Let $\pi_{1}\left(L(d, q) \times S^{1}\right)$ be generated by $\left(\bar{l}_{d}, 0\right)$ and $(0,1)$. Then $\left(\bar{l}_{d}, 0\right)$ is represented by the loop $\bar{\sigma}_{\bar{l}}=$ $\{\langle(1, t(2 \pi / d)),(0,0)\rangle, 0\}, 0 \leqq t \leqq 1$, and $(0,1)$ is represented by $\bar{\sigma}_{2}=$ $\{\langle(1,0),(0,0)\rangle, t\}, 0 \leqq t \leqq 2 \pi$. Then in $E, \sigma_{1}=\{\langle(1, t(2 \pi / d)),(0,0)\rangle, 0\}$ and $\sigma_{2}=\left\{\left\langle\left(1,-t /(2 \pi) j^{\prime} v\right),(0,0)\right\rangle, t\right\}$ represent $\left(\bar{l}_{d}, 0\right)$ and $(0,1)$ respectively. $\quad \bar{l}_{p}$ is represented by the loop $\left.\gamma=\langle(1, t v),(0,0)\rangle\right\rangle \leqq t \leqq 1$. Now the projection map $\pi: E \rightarrow L(p, q)$ is given by

We have

$$
\pi\left\{\left\langle\left(r_{1}, \theta_{1}\right),\left(r_{2}, \theta_{2}\right)\right\rangle, t\right\}=\left\langle\left\langle\left(r_{1}, \theta_{1}\right),\left(r_{2}, \theta_{2}\right)\right\rangle .\right.
$$

$$
\pi \circ \sigma_{1}=\left\langle\left\langle\left(1, t \frac{2 \pi}{d}\right),(0,0)\right\rangle\right\rangle \quad 0 \leqq t \leqq 1=\left\langle\left(1, t p^{\prime} v\right),(0,0)\right\rangle .
$$


Hence

$$
\pi_{\sharp}\left(\bar{l}_{d}, 0\right)=\bar{p}^{\prime}
$$

Also

$$
\left.\pi \circ \sigma_{2}=\left\|\left(1,-\frac{t}{2 \pi} j^{\prime} v\right),(0,0)\right\|\right\rangle \quad 0 \leqq t \leqq 2 \pi
$$

so

$$
\pi_{\#}(0,1)=-\bar{j}^{\prime} .
$$

One fiber in $E$ consists of

$$
\bigcup_{\substack{n=0 \\ 0 \leqq \pm 2 \pi}}^{p^{\prime}}\left\{\left\langle\left(1, n j^{\prime} v\right),(0,0)\right\rangle, t\right\} \text {. }
$$

Hence, in $L(d, q) \times S^{1}$, this fiber is

$$
\bigcup_{\substack{n=0 \\ 0 \leqq t \leqq 2 \pi}}^{p^{\prime}}\left\{\left\langle\left(1,\left(n+\frac{t}{2 \pi}\right) j^{\prime} v\right),(0,0)\right\rangle, t\right\}=\left\{\left\langle\left(1, \tau j^{\prime} v\right),(0,0)\right\rangle, \overline{2} \bar{\tau} \bar{\pi}\right\}
$$

where $0 \leqq \tau \leqq p^{\prime}$ and $\overline{2} \bar{\pi} \bar{\tau}$ represents the equivalence class of $2 \pi \tau(\bmod 2 \pi)$. Hence $i_{\sharp}(1)=\left(\bar{j}^{\prime}, p^{\prime}\right)$.

We have the following commutative diagram:

$$
\begin{gathered}
0 \longrightarrow \pi_{1}\left(S^{1}\right) \stackrel{i_{\sharp}}{\longrightarrow} \pi_{1}\left(L(d, q) \times S^{1}\right) \stackrel{\pi_{\sharp}}{\longrightarrow} \pi_{1}(L(p, q)) \longrightarrow 0 \\
\left(1-f_{b \sharp}\right) \\
0 \longrightarrow \pi_{1}\left(S^{1}\right) \stackrel{i_{\sharp}}{\longrightarrow} \pi_{1}\left(L(d, q) \times S^{1}\right) \stackrel{\pi_{\sharp}}{\longrightarrow} \pi_{1}(L(p, q)) \longrightarrow 0 .
\end{gathered}
$$

We must compute the cokernel of $\left(1-f_{\sharp}\right)$ since $N(f)=\mid$ coker $\left(1-f_{\sharp}\right) \mid$. Let

$$
\left(1-f_{\sharp}\right)\left(\bar{l}_{d}, 0\right)=(\bar{a}, 0)-\left(1-f_{\sharp}\right)(0,1)=(\bar{s}, u) .
$$

Commutativity of the right hand square implies that $a=1-c_{1}$, while commutativity of the left hand square implies $u=1-c_{2}$. Now

$$
\begin{aligned}
& \left(1-f_{\sharp}^{\prime}\right) \circ \pi_{\sharp}(0,1)=\overline{-\left(1-c_{1}\right) j^{\prime}} \\
& \pi_{\sharp} \circ\left(1-f_{\sharp}\right)(0,1)=\overline{p^{\prime} s-j^{\prime} u}=\overline{p^{\prime} s-j^{\prime}\left(1-c_{2}\right)} .
\end{aligned}
$$

Hence

$$
p^{\prime} s-j^{\prime}\left(1-c_{2}\right) \equiv-\left(1-c_{1}\right) j^{\prime}(\bmod p)
$$

Therefore,

$$
j^{\prime}\left(c_{2}-c_{1}\right)+p^{\prime} s=k p .
$$

We must have $p^{\prime} \mid j^{\prime}\left(c_{2}-c_{1}\right)$ so 


$$
s=k d+\frac{j^{\prime}}{p^{\prime}}\left(c_{1}-c_{2}\right) .
$$

Hence we may assume

$$
s=\frac{j^{\prime}}{p^{\prime}}\left(c_{1}-c_{2}\right)=\frac{j}{p}\left(c_{1}-c_{2}\right) .
$$

Therefore, $\operatorname{Im}\left(1-f_{\sharp}\right)$ is generated by $\overline{\left(1-c_{1}, 0\right)},(\bar{s}, 0)$, and $\left(0,1-c_{2}\right)$. Now the group $\pi_{1}\left(L(d, q) \times S^{1}\right) \simeq z_{d} \oplus z$, and the subgroup generated by $\overline{\left(1-c_{1}, 0\right)}$ and $(\bar{s}, 0)$ is the subgroup generated by $\left.\overline{\left(\left(1-c_{1}, s\right)\right.}, 0\right)$. Consequently, the cokernel of $\left(1-f_{\sharp}\right)$ is isomorphic to $z_{d} /\left(1-c_{1}, s\right) z_{d} \oplus$ $z /\left(1-c_{2}\right) z$. Which, in turn, is isomorphic to $z_{\left(d, 1-c_{1}, s\right)} \oplus z_{\left(1-c_{2}\right)}$. Therefore,

$\left|\operatorname{coker}\left(1-f_{\sharp}\right)\right|=N(f)=\left(d, 1-c_{1}, s\right) \cdot\left|1-c_{2}\right|=\left(d, 1-c_{1}, s\right) \cdot N\left(f_{b}\right)$.

Note. (1) Since $\mathscr{T}$ is orientable and $T\left(\pi^{-1}(b), e_{0}\right)=\pi_{1}\left(\pi^{-1}(b), e_{0}\right)$, the above formula is independent of $w$ and $b$.

(2) In the above argument we could replace $L(p, q)$ with the generalized lens space as in [5].

(3) If $p$ is a prime the product formula follows from results of R. Brown and E. Fadell [3].

(4) Theorem 8 also indicates that a product theorem of the type obtained by R. Brown and E. Fadell is hard to expect in general.

Corollary 9. Let $\mathscr{T}$ be as in Theorem 8. Suppose $f: E \rightarrow E$ is a bundle map such that for some $b \in L(p, q) f_{b}: \pi^{-1}(b) \rightarrow \pi^{-1}(b)$ is homotopic to a fixed-point free map. Then there exists a map $g: E \rightarrow E$, homotopic to $f$, which is fixed-point free.

Proof. Let $\tilde{f}_{b}$ be the fixed-point free map on $\pi^{-1}(b)$ which is homotopic to $f_{b}$. Clearly $N\left(\widetilde{f}_{b}\right)=0$ and since the Nielsen number is a homotopy invariant, $N\left(f_{b}\right)=0$. Thus from Theorem $8, N(f)=0$, and the corollary follows from the converse of the Lefschetz fixedpoint theorem of F. Wecken [8].

\section{REFERENCES}

1. R. F. Brown, The Lefschetz Fixed-Point Theorem, Scott, Foresman and Co., 1971.

2. - The Nielsen number of a fiber map, Ann. of Math., 85 (1967), 483-493.

3. R. F. Brown and E. R. Fadell, Corrections to "The Nielsen number of a fiber map," Ann. of Math., 95 (1972), 365-367.

4. P. J. Hilton and U. Stammbach, A Course in Homological Algebra, Springer-Verlag, 1971.

5. S. K. Kim, D. McGavran and J. Pak, The Nielsen numbers and fiberings, (to appear).

6. N. Steenrod, The Topology of Fiber Bundles, Princeton University Press, 1951.

7. M. Thornton, Total spaces of circle bundles over lens spaces, Portugale Math., (to appear). 
8. F. Wecken, Fixpunktklassen, III, Math. Ann., 118 (1942), 544-577.

Received August 29, 1973. Dennis McGavran was supported by a National Science Foundation traineeship. The authors thank the referee for his suggestions which have improved the present paper.

UNIVERSITY OF CONNECTICUT

AND

WAYNe State UNIVERSity 


\section{PACIFIC JOURNAL OF MATHEMATICS}

EDITORS

RICHARD ARENS (Managing Editor)

University of California

Los Angeles, California 90024

R. A. Beaumont

University of Washington

Seattle, Washington 98105

J. DugundJI

Department of Mathematics

University of Southern California

Los Angeles, California 90007

D. Gilbarg and J. Milgram

Stanford University

Stanford, California 94305

\section{ASSOCIATE EDITORS}

E. F. BECKENBACH

B. H. NeumanN

F. WOLF

K. YOSHIDA

\section{SUPPORTING INSTITUTIONS}

UNIVERSITY OF BRITISH COLUMBIA CALIFORNIA INSTITUTE OF TECHNOLOGY

UNIVERSITY OF CALIFORNIA

MONTANA STATE UNIVERSITY

UNIVERSITY OF NEVADA

NEW MEXICO STATE UNIVERSITY

OREGON STATE UNIVERSITY

UNIVERSITY OF OREGON

OSAKA UNIVERSITY

\author{
UNIVERSITY OF SOUTHERN CALIFORNIA \\ STANFORD UNIVERSITY \\ UNIVERSITY OF TOKYO \\ UNIVERSITY OF UTAH \\ WASHINGTON STATE UNIVERSITY \\ UNIVERSITY OF WASHINGTON \\ $\stackrel{*}{*} \stackrel{*}{*}{ }^{*}{ }^{*}{ }^{2}$ AMERICAN MATHEMATICAL SOCIETY \\ NAVAL WEAPONS CENTER
}




\section{Pacific Journal of Mathematics}

\section{Vol. 52, No. $1 \quad$ January, 1974}

David R. Adams, On the exceptional sets for spaces of potentials ............ 1

Philip Bacon, Axioms for the Čech cohomology of paracompacta ............ 7

Selwyn Ross Caradus, Perturbation theory for generalized Fredholm operators ..... 11

Kuang-Ho Chen, Phragmén-Lindelöf type theorems for a system of nonhomogeneous equations ............................ 17

Frederick Knowles Dashiell, Jr., Isomorphism problems for the Baire classes .......

M. G. Deshpande and V. K. Deshpande, Rings whose proper homomorphic images are right subdirectly irreducible . . . . . . . . . . . . . . . . . . . . . . . . .

Mary Rodriguez Embry, Self adjoint strictly cyclic operator algebras .............

Paul Erdős, On the distribution of numbers of the form $\sigma(n) / n$ and on some related

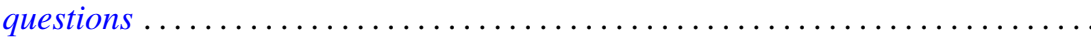

Richard Joseph Fleming and James E. Jamison, Hermitian and adjoint abelian

operators on certain Banach spaces ............................

Stanley P. Gudder and L. Haskins, The center of a poset .................. 85

Richard Howard Herman, Automorphism groups of operator algebras . . . ........

Worthen N. Hunsacker and Somashekhar Amrith Naimpally, Local compactness of families of continuous point-compact relations ....................

Donald Gordon James, On the normal subgroups of integral orthogonal groups ....

Eugene Carlyle Johnsen and Thomas Frederick Storer, Combinatorial structures in

loops. II. Commutative inverse property cyclic neofields of prime-power

order.

Ka-Sing Lau, Extreme operators on Choquet simplexes . . . . . . . . . . . . . . 129

Philip A. Leonard and Kenneth S. Williams, The septic character of 2, 3, 5 and $7 \ldots 143$

Dennis McGavran and Jingyal Pak, On the Nielsen number of a fiber map ........ 149

Stuart Edward Mills, Normed Köthe spaces as intermediate spaces of $L_{1}$ and

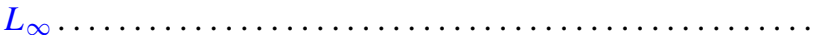

Philip Olin, Free products and elementary equivalence. .

Louis Jackson Ratliff, Jr., Locally quasi-unmixed Noetherian rings and ideals of the principal class.

Seiya Sasao, Homotopy types of spherical fibre spaces over spheres ...

Helga Schirmer, Fixed point sets of polyhedra ...

Kevin James Sharpe, Compatible topologies and continuous irreducible

representations.

Frank Siwiec, On defining a space by a weak base . . . . . . . . . . . . . . . 233

James McLean Sloss, Global reflection for a class of simple closed curves ....... 247

M. V. Subba Rao, On two congruences for primality . .

Raymond D. Terry, Oscillatory properties of a delay differential equation of even

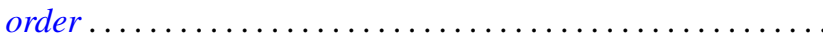

Joseph Dinneen Ward, Chebyshev centers in spaces of continuous functions . .

Robert Breckenridge Warfield, Jr., The uniqueness of elongations of Abelian

groups...

V. M. Warfield, Existence and adjoint theorems for linear stochastic differential

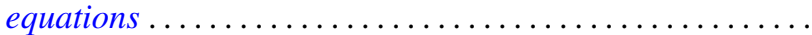

